\title{
Loss of miR-124 and miR-137 Expression in Glioblastoma Multiforme and Their Roles in Glioma Cell Proliferation and Differentiation
}

\author{
Hao $\mathrm{Wu}^{1}$, Zhenfeng Liu ${ }^{2}$, Lydia C Manor ${ }^{3^{*}}$ \\ ${ }^{1}$ Department of Neurosurgery, Xiangya 3rd Hospital, Changsha, Hunan \\ 410013, P.R. China; \\ ${ }^{2}$ Department of Neurosurgery, Xianyang Central Hospital, Xianyang, \\ Shaanxi 712000, P.R. China; \\ ${ }^{3}$ American Informatics Consultant LLC, Rockville, MD 20852, USA.
}

*Corresponding Author: Lydia C Manor, Email: I.manor@gousinfo. com.

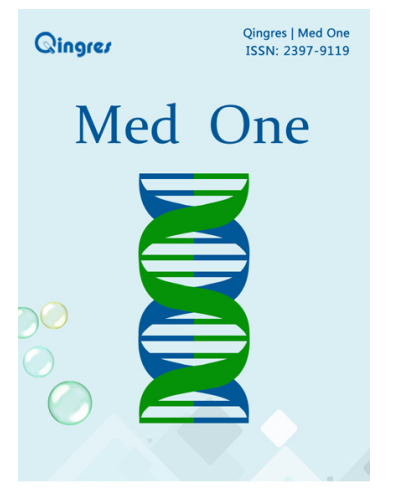

http://mo.qingres.com

\section{GOPEN ACCESS}

DOI: $10.20900 / \mathrm{mo} .20160007$

Received: January 11, 2016

Accepted: February 15, 2016

Published: April 25, 2016

Copyright: $\odot 2016$ Cain et al. This is an open access article distributed under the terms of the Creative Commons Attribution License, which permits unrestricted use, distribution, and reproduction in any medium, provided the original author and source are credited.

\begin{abstract}
Background: Glioblastoma Multiforme (GBM) is a common malignant brain tumor. It is characterized by rapid growth and high tumor heterogeneity. The molecular mechanisms driving GBM tumorigenesis and progression are not yet fully elucidated.
\end{abstract}

Methods: miR-124, and miR-137, expression in 43 high-grade GBM tumor tissues was measured by real-time PCR and compared with expression in 24 normal brain tissues. The effects of miR-124, and miR-137, overexpression on glioma U251 cell proliferation and differentiation were analyzed. Tuj1 and GFAP expression was detected using immunofluorescence staining. Cell proliferation was detected with flow cytometry.

Results: miR-124, and miR-137, expression was significantly decreased in GBM tumor tissues compared to normal brain tissues $(p<0.01)$. Immunofluorescence showed, after miR-124 and miR137 transfection, normal synapse growth structure in GBM, and that neuronal differentiation factor expression significantly increased, including Tuj1 and GFAP. Flow cytometry analysis showed that GBM cell cycle extension and differentiation was repressed.

Conclusion: miR-124, and miR-137, function as tumor suppressors to inhibit cell proliferation and differentiation and in GBM, their expression was significantly decreased. 
Key Words: Glioblastoma Multiforme; miR-124; miR-137; Proliferation; Differentiation

\section{INTRODUCTION}

Glioblastoma Multiforme (GBM) is a common malignant tumor, accounting for $80 \%$ of adult, unknown etiology, primary malignant brain tumors ${ }^{[1]}$. GBM is divided, using its pathological and clinical features, into astrocytoma, oligodendroglioma, oligoastrocytomas, and ependymoma ${ }^{[2]}$. GBM is characterized by rapid growth, high tumor heterogeneity, and diffuse infiltrative growth. These make treatment difficult and results in high recurrence, and poor survival, rates ${ }^{[3]}$. Current GBM treatment therapy is based on surgery combined with radiotherapy, chemotherapy, immunotherapy, and molecular targeting. This therapy regimen can improve GBM patient prognosis, but fails to provide a radical cure of $\mathrm{GBM}^{[4]}$. GBM cell proliferation, and differentiation, regulatory systems are of great significance to prolonging patient survival and improving prognosis.

A microRNA (miRNA) is a conserved, small, non-coding RNA molecule ( $18-25$ nucleotides long), which functions in the post-transcriptional regulation of gene expression by binding to the 3'-UTR of a target mRNA and causing subsequent cleavage, destabilization, and less efficient mRNA translation ${ }^{[5,}$ 6]. As of 2016, over 1,000 miRNAs have been found in humans, and is proposed to target about $60 \%$ of all genes ${ }^{[7,8]}$. Neural differentiation is characterized with the expression of specific proteins, including Tuj1 and GFAP, which are essential for cytoskeleton and associated with cellular morphology ${ }^{[9,10]}$. miR-124, and miR-137, overexpression results in changes in cell proliferation and differentiation ${ }^{[11]}$. Human $\beta$-Tubulin 3 is a structural protein (450 amino acid) expressed in neurons and is thought to be a neuron specific class III tubulin (Tuj1). It contributes to microtubule stability in neuronal cell bodies and axons ${ }^{[12]}$. The glial fibrillary acidic protein (GFAP) is an intermediate filament protein that expresses in numerous cell types of the central nervous system, including astrocytes and ependymal cells ${ }^{[13]}$. GFAP is involved in many important CNS processes, including astrocyte-neuron interactions, cell-cell communication, blood brain barrier function maintenance, and CNS injury repair ${ }^{[14,15]}$. This study investigated miR-124, and miR-137, expression in GBM and their effects on proliferation and differentiation, and then examined Tuj1 and GFAP expression in GBM cells.

\section{MATERIALS AND METHODS}

\subsection{Sample collection}

A total of 43 high-grade GBM tumor tissue samples were collected, between December 2012 and December 2014, from patients before chemotherapy, radiotherapy, or other treatments. GBM was diagnosed using head MRI and pathological examinations. Of the 43 cases, 23 were male and 20 were female with an average age of $39.1 \pm$ 6.5 . There were 24 relatively normal brain tissues collected around the trauma of cerebral internal injuries as controls. Of the 24 controls, 13 were male and 11 were female with an average age of $41.3 \pm 8.2$. Tumor, and control brain, tissues were obtained during surgery and immediately placed in liquid nitrogen. This study was approved by ethics committee. Signed consent forms were collected from all study subjects.

\subsection{Cell culture}

The U251 glioma cell line was provided by the Chinese Academy Sciences Cell Bank (China) and cultured, at $37^{\circ} \mathrm{C}, 5 \% \mathrm{CO}_{2}$, in an RPMI 1640 medium containing $10 \%$ fetal bovine serum (FCS), $4 \times$ nonessential amino acids, $3 \mathrm{mM} \mathrm{L}$-Glutamine, Penicillin (100 U/ml), and, Streptomycin (100 mg/ml).

\subsection{Real-time RT-PCR}

miR-124, and miR-137, expression levels in GBM tumor tissues and normal brain tissues were detected by using a Real-time RT-PCR. First, primers were designed based on an miR-124 and miR-137 sequence (GeneBank accession numbers: NR_0296 68, and NR_029679, respectively) (Table 1). RNA was extracted from tissues with an RNAprep Pure Tissue Kit. Real-time RT-PCR was performed by a Real-time PCR Amplifier (Bio-Rad) using an mirVanat qRT-PCR miRNA kit (Ambion) under the following reaction conditions: $95^{\circ} \mathrm{C}$, for $3 \mathrm{~min} ; 95^{\circ} \mathrm{C}$ for $15 \mathrm{~s}$; and $60^{\circ} \mathrm{C}$ at $30 \mathrm{~s}$ for 40 cycles. U6 was used as a reference gene. Results were analyzed using $2-\Delta \Delta \mathrm{Ct}^{[16]}$. 
Table 1. Synthesis of nucleic acid sequence 2 for cell transfection

\begin{tabular}{lll}
\hline Name & Sequence & Tm( $\left.{ }^{\circ} \mathbf{C}\right)$ \\
\hline miR-124-F & 51.8 \\
miR-124-R & 5' AGGCCTCTCTCTCCGTG 3' & 51.2 \\
miR-137-F & 5' GGTCCTCTGACTCTCTTC3' & 51.5 \\
miR-137-R & 5' TGCCGCTGGTACTCTCC 3' & 53.0 \\
\hline
\end{tabular}

\subsection{Cell transfection}

Matured miR-124, and miR-137, sequences were used to establish an miR-124, and miR137, overexpression system. The corresponding sequences with the same oligonucleotide sequences compositions were used as negative controls. miR124, and miR-137, sequences were synthesized by the Nanjing Shengxing Company. After cloning the synthesized miRNA sequences into the pMIRREPORT expression system (Ambion), the plasmid DNA with correct insertion WAS transfected into U251 glioma cells using INTERFERinTM Polyplus transfection reagents. THE U251 GBM cell line was purchased from the Cell Bank of the Chinese Academy of Science. Cells were cultured in an RPMI-1640 medium (HyClone) at $37^{\circ} \mathrm{C}$ and $5 \%$ $\mathrm{CO}_{2}$ for $24 \mathrm{hrs}$ after transfection. Cells were then subjected to further analysis.

\subsection{Imunofluorescence}

Immunofluorescence was used to detect Tuji and GFAP expression levels in miRNA-expressionvector-transfected U251 cells. Logarithmic growth phase transfected cells were fixed with paraffin onto slides. After blocking, cells were incubated with mouse anti-human an Tuji and GFAP (1:2000) antibody for $2 \mathrm{hrs}$ at $37^{\circ} \mathrm{C}$, followed by incubation with a fluorescence-marked goat anti-mouse secondary antibody $(1: 1000)$ for $1 \mathrm{hr}$ at $37^{\circ} \mathrm{C}$. After washing with $1 \times \mathrm{PBS}$ and attaching cover slips to the slices, fluorescent staining was observed under a fluorescence microscope.

\subsection{Flow cytometry assay}

miR-124 and miR-137-transfected U251 glioma cells were grown to the logarithmic phase (24 hrs) and fixed with $90 \%$ ethanol, overnight, at $4^{\circ} \mathrm{C}$. After removing the ethanol, cells were incubated with RNase for $30 \mathrm{~min}$ at $37^{\circ} \mathrm{C}$ and cell nuclei were stained with $\mathrm{PI}$. The stained cells were then subjected to a flow cytometry assay (Becton Dickinson) with an excitation wavelength of $488 \mathrm{~nm}$ and an emission wavelength of $630 \mathrm{~nm}$. The FL-2 area, and DNA histograms, were analyzed by Modifit software. All experiments were repeated 3 times.

\subsection{Statistical Analysis}

Results were analyzed using SPSS 20.0 and presented with a mean \pm standard deviation. $T$ test was used for comparative analysis. $p<0.05$ was deemed as statistically significant.

\section{RESULTS}

\section{1 qRT-PCR of miR-124 and miR-137 expression in GBM tissues}

miR-124, and miR-137, expressions in GBM was examined with RT-PCR (Fig. 1). miR-124, and miR-137, expression in GBM tissues significantly decreased compared to normal brain tissue ( $p$ $<0.01$ ). miR-124, and miR-137, expression in normal brain tissue was 12 and 15 times higher, respectively, than that in GBM tumor tissues. 


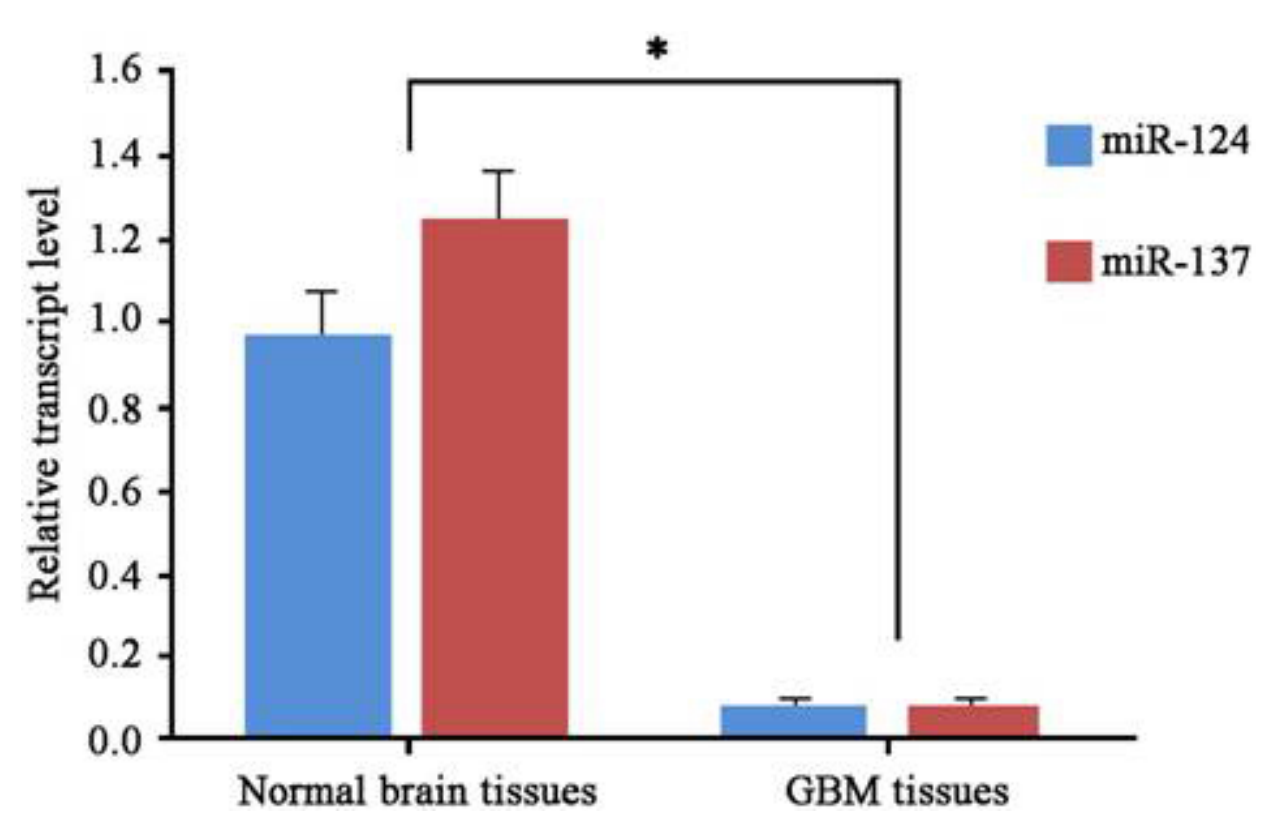

Fig. 1 Relative expression of miR-124 and miR-137. miR-124 and miR-137 expression in normal brain tissues and GBM tumor tissues was measured by real-time PCR. ${ }^{*} p<0.01$ between normal brain tissues and GBM tumor tissues.

3.2 Overexpression of $\mathrm{miR}-124$ and $\mathrm{miR}-$

\section{7}

A pMIR-REPORT expression system was used to detect miR-124, and miR-137, overexpression in U251 glioma cells. Total RNA was extracted. miR-
124, and miR-137, expression was measured using real-time PCR. miR-124 and miR-137, relative expression is shown in Fig. 2. Cell transfection significantly increased miR-124, and miR-137, expression in U251 glioma cells compared to control cells $(p<0.05)$.

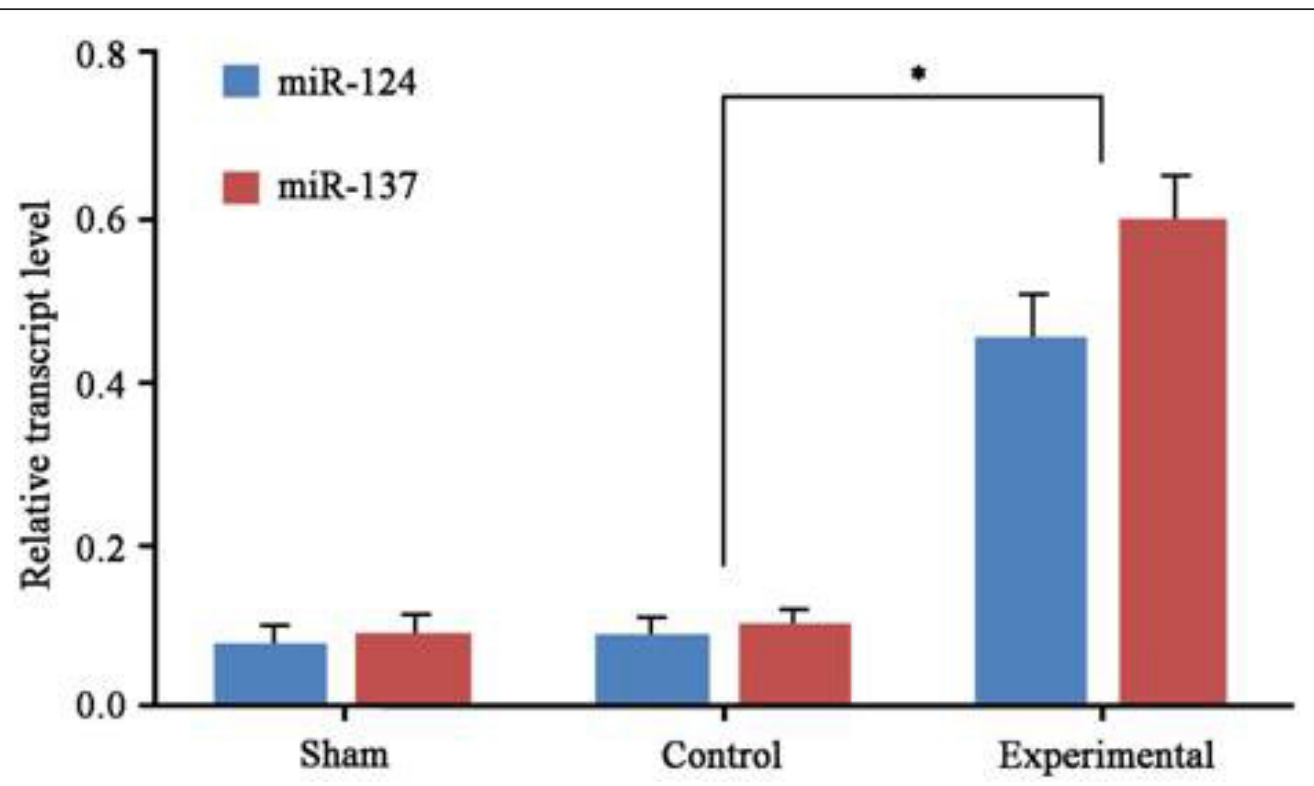

Fig. 2 Overexpression of miR-124 and miR-137 in U251 cells. Glioma U251 cells were trransfected with miR124 and miR-137 expression vector, negative control vector (control), and without vector (sham). 24 hrs after the transfection, miR-124 and miR-137 expression in transfected cells were measured by real-time PCR. $p<0.05$ between miR-124 or miR-137 trransfected cells and control cells. 


\subsection{Immunofluorescence of Tuj1 and GFAP expression}

miR-124, and miR-137, transfected U251 cells were harvested every other day. Tuj1, and GFAP, expression was detected by immunofluorescence staining (Fig. 3). Tuj1 was shown with GFP (a green fluorescent marker), GFAP with rhodamine (a red fluorescent marker). Tuj1 and GFAP cellular expression levels represent the degree of cell differentiation as specific proteins of the neuron system. With cell culture progression, Tuj1, and GFAP, expression gradually increased after miR124, and miR-137, transfection. Phase contrast microscopy showed neuron-specific synapse structures in U251 cells after miR-124, and miR137, overexpression, suggesting that the U251 cells differentiated into normal neurons.

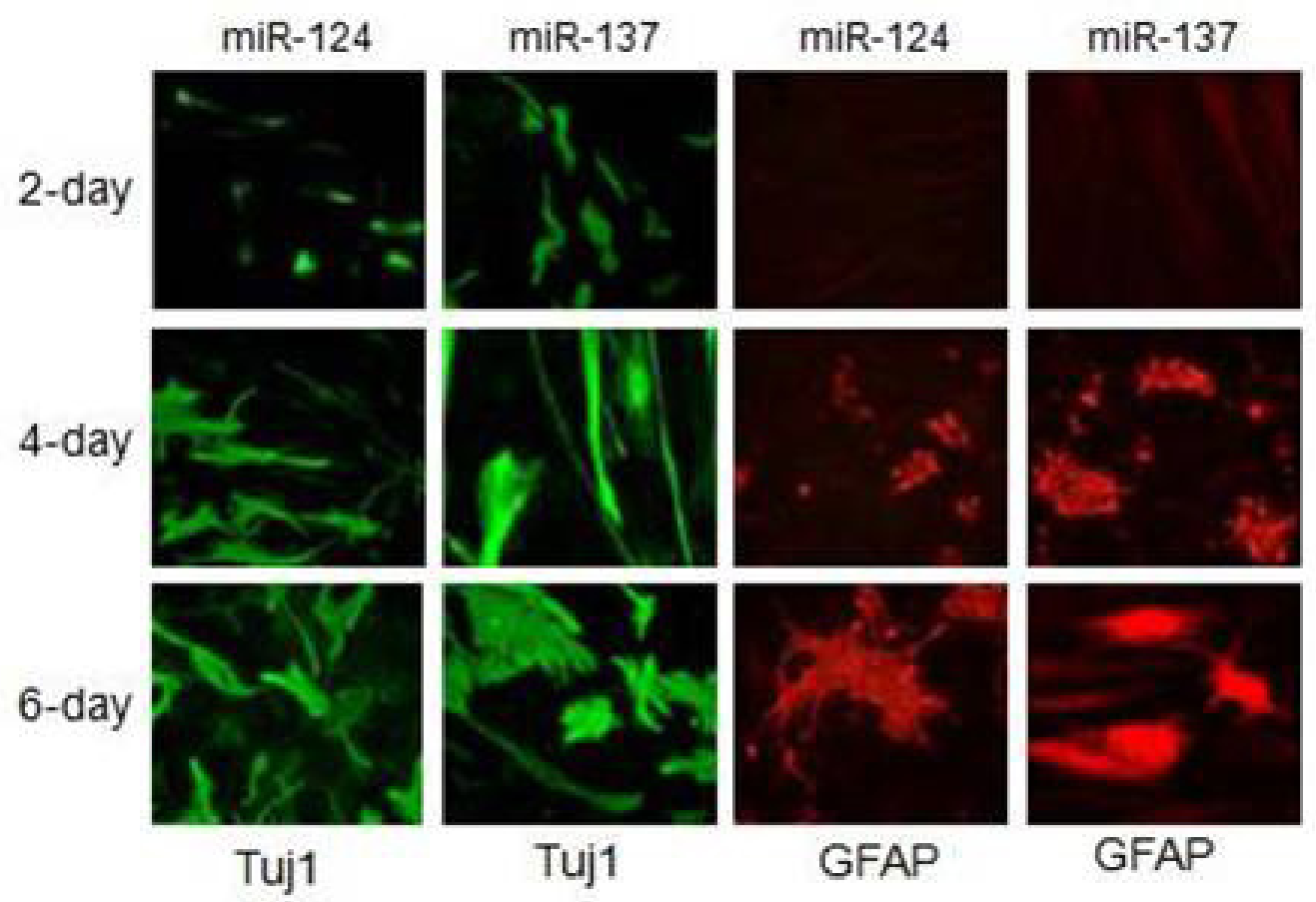

Fig. 3

Fig. 3 Immunofluorescence of Tuj1 and GFAP expression. U251 cells were transfected with miR-124 or miR137 expression vectors. Tuj1 (green) and GFAP (red) expression was detected by immunofluorescence staining (400x). A time-dependent increase in fluorescence intensity (expression level) was observed.

\subsection{Flow cytometry analysis of the cell cycle}

The cell growth cycle was measured using the flow cytometry of miR-124, and miR-137, which had transfected, blank-controlled, and negative-controlled U251 cells. FL-2 areas were analyzed using Modifit software (Fig. 4A). Cell distribution in different cell cycle stages was analyzed using SPSS 20.0 software (Fig. 4B, 4C). No differences were observed between: blank, and negative, G0/G1 phase control cells; S phase; or G2/M phase ( $p>0.05)$. miR-124, and miR-137, transfection significantly increased the number of U251 cells in the G0 / G1 phase and decreased the number of U251 cells in the $S$, and $\mathrm{G} 2 / \mathrm{M}$, phases $(p<0.05)$, suggesting that cell cycle was prolonged and mitosis attenuated. 

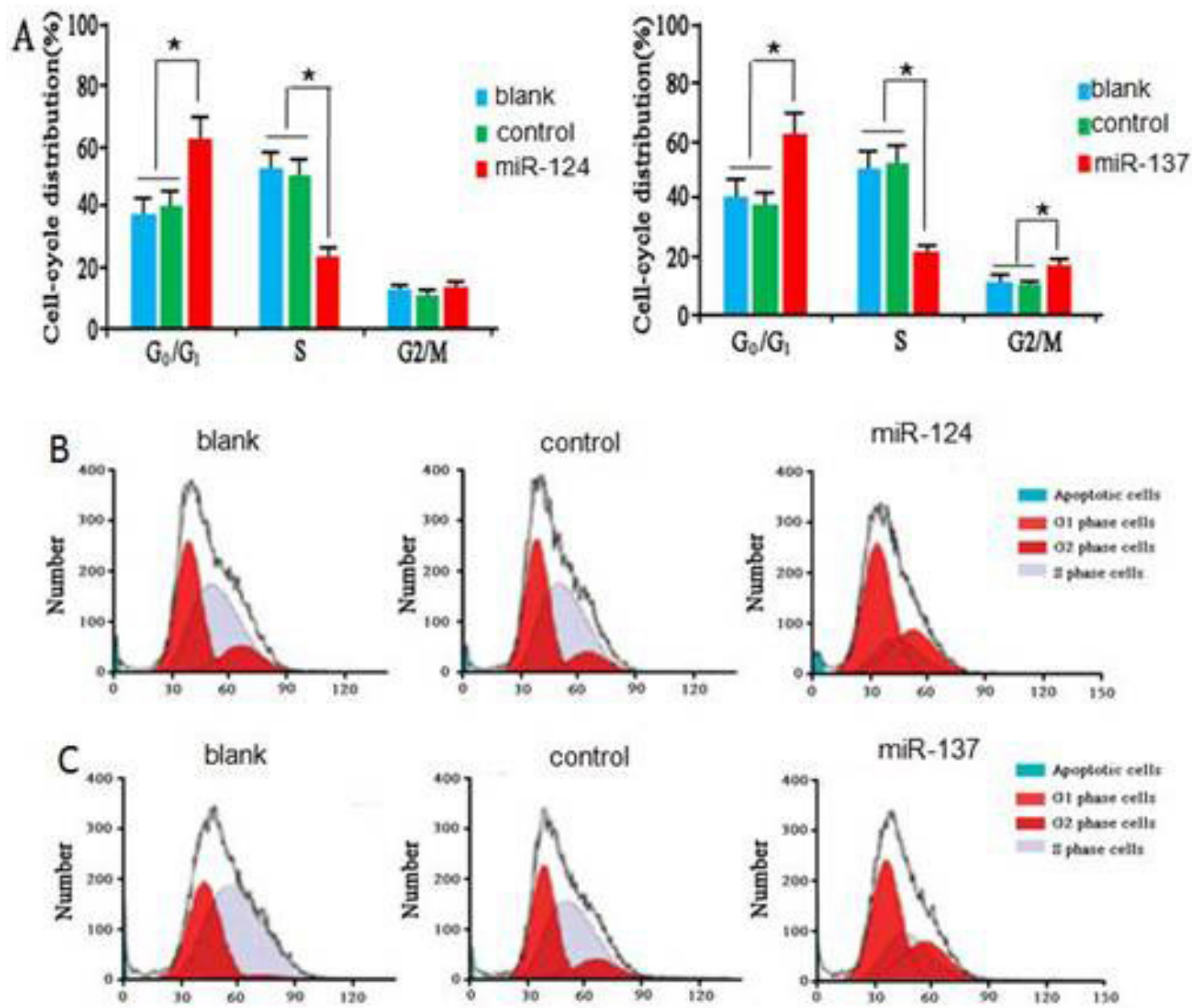

Fig. 4

Fig. 4 Flow cytometry assay of cell cycle of miR-124 and miR-137 overexpressed U251 cells. (A) Cell cycle distribution. ${ }^{*} p<0.05$ between miR-124 or miR-137 trransfected cells and control cells. (B) Representative photogram of the number of cells in each cell cycle in U251 cells transfected with miR-124 vector, negative vector, and without vector. (C) Representative photogram of cell cycle distribution in U251 cells transfected with miR-137 vector, negative vector, and without vector.

\section{DISCUSSION}

This study first found significantly decreased miR124, and miR-137, expression in high-grade GBM tumor tissues. Further investigation of glioma U251 cells, using an established overexpression model, confirmed miR124, and miR-137, effects in cell proliferation. Immunofluorescence staining revealed that miR124 and miR-137 overexpression stimulated neuronal differentiation. miR-124, and miR-137, abnormalities have been previously reported in GBM. But their biological functions have not been fully elucidated. Santos et al. demonstrated that low miR-124, and miR-137, expression is associated with GBM cell proliferation by maintaining activation of growth factors, such as EGF and FGF ${ }^{[17]}$. miR124 and miR-137 decreased expression in GBM is proposed to result from irreversible modification of transcription regulation. For example, Chakrabarti et al. showed that miR-124 expression level was elevated in GBM after a DNA demethylation (hypomethylation) modifier, 5-Aza-2-deoxycytidine 
treatment ${ }^{[18]}$. This study provided no evidence as to the cause of low miR-124, and miR-137, expression in GBM tumor tissues, it demonstrated that miR-124, and miR-137, expression was significantly lowered in high grade GBM tumor tissues. Cell culture experiments with artificial expressions of these two miRNAs significantly inhibited glioma U251 cell proliferation. This study suggests that both miR124, and miR-137, are GBM tumor suppressors. The roles of miR-124 and miR-137 in neuronal differentiation have been previously observed. miR124 overexpression can enhance embryonic stem cell differentiation into neurons ${ }^{[19]}$. miR-124 was found to regulate PTBP1 expression, which plays a role in inhibiting alternative splicing patterns of mRNA precursors in non-neuronal cells ${ }^{[20]}$. Research has also found that miR-124 promotes neuronal differentiation by inhibiting SCP1 expression ${ }^{[21]}$. miR-137 was reported to inhibit CDK6 expression and subsequently participate in cell cycle, and mitosis, regulation ${ }^{[22]}$. This study found that miR124 and miR-137 overexpression promotes U251 glioma cells differentiation into normal neurons and

\section{REFERENCES}

1. Deng X, Ma L, Wu M, Zhang G, Jin C, Guo Y, Liu R. miR-124 radiosensitizes human glioma cells by targeting CDK4. J Neurooncol. 2013; 114(3): 263-274.

2. Huse JT, Holland EC. Targeting Brain Cancer: advances in the molecular pathology of malignant glioma and medulloblastoma. Nat Rev Cancer. 2010; 10(5): 319-331.

3. Stupp R, Hegi ME, Mason WP, van den Bent MJ, Taphoorn MJ, Janzer RC. Effects of radiotherapy with concomitant and adjuvant temozolomide versus radiotherapy alone on survival in glioblastoma in a randomised phase III study: 5-year analysis of the EORTC-NCIC trial. Lancet Oncol. 2009; 10(5): 459-466.

4. Friedman HS, Prados MD, Wen PY, Mikkelsen T, Schiff D, Abrey LE, Vredenburgh J. Bevacizumab alone and in combination with irinotecan in recurrent glioblastoma. J Clin Oncol. 2009; 27(28): 4733-4740.

5. Baraniskin A, Kuhnhenn J, Schlegel U, Maghnouj A, Zöllner H, Schmiegel W, Schroers R. Identification of microRNAs in the cerebrospinal fluid as biomarker for the diagnosis of glioma. Neuro Oncol. 2012; 14(1): 29-33. is accompanied by Tuj1, and GFAP, expression elevation.

As a severe brain tumor, GBM threatens human health, making diagnosis of, and treatment for, GBM an issue of common concern in clinical scenarios $^{[1]}$. The instant study proved that miR124 and miR-137 expression significant decreased in GBM cells, thus providing evidence for clinical diagnosis. It was also found that miR-124, and miR137, over-expression induced GBM differentiation. Given that differentiation therapy is currently of great interest to the tumor field, as it induces cancer cells to transform with normal morphology, gene expression and function ${ }^{[22]}$, the findings of this study will contribute to an improvement in differentiation therapy.

\section{CONFLICT OF INTERESTS}

The authors declare no conflict of interests.

6. Fu X, Tian J, Zhang L, Chen Y, Hao, Q. Involvement of microRNA-93, a new regulator of $\mathrm{PTEN} / \mathrm{Akt}$ signaling pathway, in regulation of chemotherapeutic drug cisplatin chemosensitivity in ovarian cancer cells. FEBS Lett. 2012; 586(9): 1279-1286.

7. Zhao $W H, W u S Q$, Zhang YD. Downregulation of miR-124 promotes the growth and invasiveness of glioblastoma cells involving upregulation of PPP1R13L. Int J Mol Med. 2013; 32(1): 101107.

8. Chen L, Wang X, Wang H, Li Y, Yan W, Han L, $\mathrm{Pu}$ P. miR-137 is frequently down-regulated in glioblastoma and is a negative regulator of Cox2. Eur J Cancer. 2012; 48(16): 3104-3111.

9. Guan S, Ge D, Liu TQ, Ma XH, Cui ZF. Protocatechuic acid promotes cell proliferation and reduces basal apoptosis in cultured neural stem cells. Toxicol in Vitro. 2009; 23(2): 201208.

10. Wong W, Farr R, Joglekar M, Januszewski A, Hardikar A. Probe-based real-time PCR approaches for quantitative measurement of microRNAs. J Visual Exp. 2015; 98: e52586. 
11. Mandir JB, Lockett MR, Phillips MF, Allawi HT, Lyamichev VI, Smith LM. Rapid determination of RNA accessible sites by surface plasmon resonance detection of hybridization to DNA arrays. Anal Chem. 2014; 81(21): 8949-8956.

12. Zhang $Y$, Yao X, Jiang C, Yue J, Guan J, Cheng $H$, Fan L. Expression of PI3K, PTEN and Akt in small intestinal adenocarcinoma detected by quantum dots-based immunofluorescence technology. Cancer Biomark. 2012; 13(4): 299305.

13. Smits M, Nilsson J, Mir SE, van der Stoop PM, Hulleman E, Niers JM, de Witt Hamer PC, Marquez VE, Cloos J, Krichevsky AM, Noske DP, Tannous BA, Würdinger T. miR-101 is down-regulated in glioblastoma resulting in EZH2-induced proliferation, migration, and angiogenesis. Oncotarget. 2010; 1(8): 710-720.

14. Lakomý R, Šána J, Hankeová $S$, Fadrus $P$, Křen L, Svoboda M, Slabý O. miR-195, miR-196b, miR-181c, miR-21 expression levels and MGMT methylation status are associated with clinical outcome in glioblastoma patients. Cancer Sci. 2011; 102(12): 2186-2190.

15. Santos MC, Tegge AN, Correa BR, Mahesula S, Kohnke LQ, Qiao M, Ferreira MA, Kokovay E, Penalva LO. miR-124, -128 , and -137 Orchestrate neural differentiation by acting on overlapping gene sets containing a highly connected transcription factor network. Stem Cells. 2016; 34(1): 220-232.

16. Chakrabarti M, Ray SK. Direct transfection of miR-137 mimics is more effective than DNA demethylation of miR-137 promoter to augment anti-tumor mechanisms of delphinidin in human glioblastoma U87MG and LN18 cells. Gene. 2015; 573(1): 141-152.

17. Shimizu T, Suzuki H, Nojima M, Kitamura $\mathrm{H}$, Yamamoto $\mathrm{E}$, Maruyama $\mathrm{R}$, Tokino $\mathrm{T}$. Methylation of a panel of microRNA genes is a novel biomarker for detection of bladder cancer. Eur Urol. 2013; 63(6): 1091-1100.

18. Neo WH, Yap K, Lee SH, Looi LS, Khandelia $\mathrm{P}$, Neo SX, Su IH. MicroRNA miR-124 controls the choice between neuronal and astrocyte differentiation by fine-tuning Ezh2 expression. J Biol Chem. 2014; 289(30): 20788-20801.

19. Li M, Plecita L, McKeon B, Flockton A, Frid M $\mathrm{G}$, Jezek P, Zhang H. miR-124 regulates the metabolic state of vascular adventitial fibroblasts in pulmonary hypertension through the Rna splicing factor Ptbp1. Am J Respir Crit Care Med. 2014; 189: A3986.

20. Franke K, Otto W, Johannes S, Baumgart J, Nitsch R, Schumacher S. miR-124-regulated RhoG reduces neuronal process complexity via ELMO/Dock180/Rac1 and Cdc42 signaling. EMBO J. 2012; 31(13): 2908-2921.

21. Deng $X$, Ma L, Wu M, Zhang G, Jin C, Guo $Y$, Liu R. miR-124 radiosensitizes human glioma cells by targeting CDK4. J Neurooncol. 2013; 114(3): 263-274.

22. Campos B, Wan F, Farhadi M, Ernst $A$, Zeppernick F, Tagscherer K E, Combs S E. Differentiation therapy exerts antitumor effects on stem-like glioma cells. Clin Cancer Res. 2010; 16(10): 2715-2728. 